Revue internationale P.M.E.

Économie et gestion de la petite et moyenne entreprise

\title{
Le responsable de PME français et québécois membre d'une association de dirigeants : quelle utilité en matière décisionnelle?
}

\section{Paméla Baillette}

Volume 16, numéro 1, 2003

URI : https://id.erudit.org/iderudit/1008432ar

DOI : https://doi.org/10.7202/1008432ar

Aller au sommaire du numéro

Éditeur(s)

Presses de l’Université du Québec

ISSN

0776-5436 (imprimé)

1918-9699 (numérique)

Découvrir la revue

Citer cet article

Baillette, P. (2003). Le responsable de PME français et québécois membre d'une association de dirigeants : quelle utilité en matière décisionnelle ? Revue internationale P.M.E., 16(1), 43-73. https://doi.org/10.7202/1008432ar
Résumé de l'article

Présentée généralement comme un moyen utile et convivial pour obtenir des informations liées à l'activité professionnelle, l'association de dirigeants n'en est pas moins mal connue quant à ses apports véritables pour l'aide à la décision.

L'exploitation des résultats d'une étude empirique menée en France et au Canada (Québec) propose une réflexion sur sa pertinence en la matière, puis sur les facteurs qui favorisent le succès de cette aide.

Complémentaire aux autres sources d'aide potentiellement mobilisables par les responsables de PME, l'association occupe néanmoins un positionnement spécifique dû à la combinaison particulière de ses caractéristiques. Reconnu principalement dans les phases amont du processus décisionnel, le succès de l'aide dépend d'une série de facteurs liés à la mission de l'association, à la structure et au fonctionnement des groupes de travail, et à la gestion de l'association. 


\title{
Le responsable de PME français et québécois membre d'une association de dirigeants: quelle utilité en matière décisionnelle?
}

Paméla BAILLETTE

Université de Perpignan

\author{
MOTS CLÉS
}

Dirigeant de PME - Réseaux associatifs

Aide à la décision - Canada (Québec)

\section{RÉSUMÉ}

Présentée généralement comme un moyen utile et convivial pour obtenir des informations liées à l'activité professionnelle, l'association de dirigeants n'en est pas moins mal connue quant à ses apports véritables pour l'aide à la décision.

L'exploitation des résultats d'une étude empirique menée en France et au Canada (Québec) propose une réflexion sur sa pertinence en la matière, puis sur les facteurs qui favorisent le succès de cette aide.

Complémentaire aux autres sources d'aide potentiellement mobilisables par les responsables de PME, l'association occupe néanmoins un positionnement spécifique dû à la combinaison particulière de ses caractéristiques. Reconnu principalement dans les phases amont du processus décisionnel, le succès de l'aide dépend d'une série de facteurs liés à la mission de l'association, à la structure et au fonctionnement des groupes de travail, et à la gestion de l'association.

\section{L'AUTEURE}

Paméla Baillette est maître de conférences en sciences de gestion à l'Université de Perpignan (JEREM) et membre associé au CREGO (Centre de recherche en gestion des organisations) à l'Université de Montpellier II. Elle a obtenu en 2000 un doctorat en sciences de gestion sous la direction du professeur Robert Reix et délivré par l'Université de Montpellier II-Institut d'administration des entreprises (IAE). Ses recherches portent sur la prise de décision, les contacts associatifs mobilisés par les dirigeants de PME et la confiance dans les échanges informationnels entre acteurs organisés en réseaux. Adresse: Université de Perpignan, 52, avenue de Villeneuve, 66860 Perpignan cedex. Courriel : pamela.baillette@voila.fr 


\begin{abstract}
Company directors' associations are generally perceived as a convivial and useful way of obtaining information related to a professional activity. They are less wellknown for their contribution in terms of assistance in decision-making.

The interpretation of an empirical study carried out in France and Canada (Quebec) forms the basis for reflection on the pertinence of such assistance and the factors which contribute to the success of this decision-making help.

Such associations are complementary to other external sources of assistance that SME managers can call upon but nevertheless occupy a specific position due to a combination of characteristics. They are primarily recognised as influential in the early stages of decision-making and the success of their intervention and assistance depends on a series of factors related to the association's mission, the way work groups meet and on how the association itself is run.
\end{abstract}

\title{
RESUMEN
}

Presentada generalmente como un medio útil y práctico para obtener información vinculada a la actividad profesional, la asociación de dirigentes no es menos conocida en cuanto a sus auténticos aportes en la ayuda a la decisión.

La explotación de los resultados de un estudio empírico realizado en Francia y en Canadá (Quebec) propone una reflexión sobre su pertinencia en la materia y luego en los factores que favorecen el éxito de esta ayuda.

Complementaria a las otras fuentes de ayuda potencialmente movilizables por los responsables de las PyMEs, la asociación ocupa sin embargo una posición específica debida a la combinación particular de sus características. Reconocido principalmente en las fases precedentes al proceso de decisiones, el éxito de la ayuda depende de una serie de factores vinculados a la misión de la asociación, a la estructura y al funcionamiento de los grupos de trabajo y a la gestión de la asociación.

\section{ZUSAMMENFASSUNG}

Im allgemeinen werden Geschäftsführervereinigungen als ein nützliches und einladendes Mittel präsentiert, um zu Informationen in der beruflichen Tätigkeit zu gelangen. Gleichzeitig sind sie nicht weniger bekannt für ihren positiven Beitrag bei der Entscheidungshilfe.

Die Auswertung der Resultate einer empirischen Studie aus Frankreich und Kanada (Québec) schlägt eine Reflexion vor über die Erheblichkeit und dann über die Faktoren, die den Erfolg dieser Hilfe beeinflussen.

Ergänzend zu anderen möglichen Quellenhilfen die durch Verantwortliche in KMU mobilisiert wurden, hat die Vereinigung nichts desto weniger eine spezielle Position, die in der besonderen Kombination von seinen Merkmalen liegt. Grundsätzlich anerkannt in den vorwärtsstrebenden Phasen des Entscheidungsprozesses, ist der Erfolg der Hilfe abhängig von einer Reihe von Faktoren die gebunden sind an die Mission der Vereinigung, an die Struktur und an die Funktionsweise der Arbeitsgruppen und an die Führung der Vereinigung.

Revue internationale P.M.E., vol. 16, nº 1, 2003 


\section{Introduction}

Activité délicate dans toutes les entreprises, la prise de décision se révèle particulièrement risquée dans les PME où les actions engagées peuvent très rapidement nuire à la pérennité de l'entreprise et souvent mettre en danger le patrimoine du dirigeant. De plus, la faiblesse relative des moyens dans ce type d'entreprises limite les possibilités d'accès aux sources d'informations mobilisées par les dirigeants d'entreprises plus importantes. Les activités relationnelles créées et entretenues à l'intérieur d'un réseau ${ }^{1}$ peuvent alors apparaître comme un moyen utile pour les décideurs dans les PME.

En tant que dirigeants, ils sont en contact avec de nombreuses personnes dans leur environnement. Certains choisissent pourtant d'adhérer et de participer à un type spécifique de réseaux que nous nommons «associations de dirigeants à finalité de réflexion et d'action sur la gestion de l'entreprise ». Leur mission consiste à aider les adhérents dans leurs pratiques de gestion grâce à une réflexion menée en commun sur des thèmes liés à l'activité professionnelle ${ }^{2}$.

L'étude présentée ici a pour objet d'évaluer le succès de l'aide apportée par l'association au plan décisionnel. Nous précisons que ce travail de recherche ne se limite pas à la prise de décision (généralement comprise comme un acte ponctuel de choix), mais qu'il englobe l'ensemble du processus décisionnel individuel, dont le choix ne représente qu'une phase particulière ${ }^{3}$. L'information collectée par le décideur peut ainsi être utilisée dans toutes les étapes du processus.

1. De nombreux acteurs manifestent actuellement de l'intérêt pour ce sujet:

- les gestionnaires, avec les efforts qu'ils engagent pour développer des liens interorganisationnels ;

- la communauté scientifique (Julien et Marchesnay, 1988 ; Curran et Blackburn, 1994 ; Duchéneaut, 1996; GREPME, 1997, notamment) qui réalise des recherches approfondies dans ce domaine;

- les pouvoirs publics, qui encouragent aujourd'hui fortement la coopération interentreprises.

2. Duchéneaut (1996) cite à titre d'exemple le « CJD» (Centre des jeunes dirigeants d'entreprise), «l'APM » (Association progrès du management), «Entreprise et progrès », «ETHIC» (Entreprise de taille humaine indépendante et de croissance), «Institut de l'entreprise », etc. Voir Marty et Ivanoff (1996) pour une liste détaillée des réseaux fréquentés par les dirigeants d'organisations.

3. Selon Reix (1995) et Trahand (1999), l'aide à la décision peut se réaliser aux différentes phases du processus décisionnel individuel (Simon, 1965; Mintzberg et al., 1976; Nutt, 1993a et b), en l'occurrence à la phase d'intelligence (reconnaissance et compréhension de stimuli), à la phase de modélisation (développement de une ou plusieurs solutions au problème posé), à la phase de choix (sélection parmi les solutions possibles) et à la phase de mise en œuvre de la solution choisie. Ce modèle contient des limites qui portent principalement sur l'ordre et l'enchaînement des phases du processus, et sur le principe de rationalité humaine, mais les détails qu'il fournit permettront de préciser la façon dont l'association peut enrichir le processus décisionnel des dirigeants de PME membres.

Revue internationale P.M.E., vol. 16, nº 1, 2003 
Dans ce contexte, nous définissons le «succès» de l'aide par référence au modèle des « $3 \mathrm{~F} »{ }^{4}$ qui prend en compte, en situation de prise de décision, les critères d'efficacité, d'efficience et d'effectivité (Paturel, 2000). Ainsi, l'aide est perçue par l'adhérent comme étant :

- efficace, si l'association l'a aidé à atteindre les buts (objectifs) qu'il s'était fixés ;

- efficiente, si l'adhérent a réalisé des économies de moyens (matériels, humains, financiers, d'informations, de temps, etc.) : l'aide obtenue lui a permis d'utiliser moins de ressources qu'il n'en aurait consommées s'il avait mobilisé d'autres sources d'aide à la décision (pour obtenir un résultat similaire), ou bien l'aide lui a permis d'améliorer les résultats acquis pour un même investissement (matériel ou immatériel);

- effective ${ }^{5}$, si l'adhérent est satisfait de la contribution apportée par ses pairs sur la base de l'évaluation des résultats obtenus.

Les travaux scientifiques consacrés aux associations qui développent des thèmes de réflexion sur la gestion de l'entreprise sont relativement réduits, même si certaines publications récentes ont mis l'accent sur ce sujet (en particulier l'ouvrage de B. Duchéneaut, 1996). Le manque d'éléments précis sur le rôle joué en matière décisionnelle par ces associations nous a conduite à mener une étude sur le terrain. Cette étude tente de répondre à deux questions successives de recherche visant, pour la première, à vérifier que l'association est bien perçue par les responsables de PME comme un moyen capable d'apporter une aide à leur processus décisionnel individuel et, pour la seconde, à faire émerger les facteurs qu'ils perçoivent comme étant favorables au succès de cette aide.

Nous signalerons tout d'abord l'importance de l'activité relationnelle pour les décideurs de PME, en nous centrant sur le rôle théoriquement joué dans l'aide à la décision par les associations de dirigeants. Nous exposerons ensuite la

4. L'EFficacité (premier F) concerne le respect des objectifs assignés, l'EFficience (deuxième $\mathrm{F}$ ) vise la réalisation des extrants en respectant les normes d'utilisation d'intrants en la matière; l'obtention d'une bonne EFfectivité (troisième F) permet de s'assurer que le personnel se trouve dans de bonnes conditions de travail et de vie ainsi que dans une bonne ambiance générale (Paturel, 2000, p. 178).

5. Pour certains auteurs, au-delà de la recherche d'une amélioration constante de l'efficience, la performance comprend également les notions d'efficacité et de satisfaction, ou d'effectivité (Marchesnay, 1988; Birley et Westhead, 1990 ; Guillon et Halley, 1996) laquelle suppose de répondre aux besoins et aux exigences des membres de l'organisation (Friedlander et Pickle, 1968). Ainsi, l'efficience, l'efficacité et l'effectivité constitueraient les facteurs explicatifs de la performance (Guillon et Halley, 1996).

Revue internationale P.M.E., vol. 16, nº 1, 2003 
méthodologie choisie pour répondre aux questions de recherche. Les résultats pourront alors être exposés et détaillés afin de préciser l'apport de l'association en matière décisionnelle.

\section{Fondements théoriques}

Le contexte décisionnel spécifique de la PME suscite l'intérêt pour son dirigeant de créer et d'entretenir un réseau personnel de contacts. À l'intérieur de ce réseau, nous étudierons la place occupée par l'association de dirigeants en la comparant avec d'autres sources potentiellement mobilisables par les responsables d'entreprise.

\subsection{Contexte décisionnel de la PME}

Par rapport aux grandes entreprises, le contexte de la prise de décision dans les PME présente deux caractéristiques principales. La première est liée à l'autonomie importante et au sentiment de solitude décisionnelle ressenti par le responsable de PME. La seconde est relative aux conséquences rapides des décisions prises sur la situation personnelle du dirigeant, d'une part, et sur celle de l'entreprise, d'autre part.

Le dirigeant de PME occupe ainsi une position clé dans l'entreprise. L'étendue de son pouvoir décisionnel se révèle être d'autant plus importante que la taille de l'entreprise est petite. Les auteurs spécialisés dans l'étude des petites entreprises soulignent généralement la place centrale occupée par le dirigeant (Marchesnay, 1982 ; d'Amboise et Muldowney, 1988; Julien et Marchesnay, 1988; Duchéneaut, 1995, 1996). Parmi les caractéristiques principales de la petite entreprise, Julien et Marchesnay (1988) retiennent comme critère fondamental la centralisation sinon la personnalisation de la gestion. Ce phénomène se manifeste d'autant plus fortement qu'on assiste souvent à une assimilation de la propriété du capital au pouvoir de gestion (Fallery, 1983; Van Loye, 1991). Cette personnalisation conduit à des décisions qui reposent sur l'expérience et sur les connaissances personnelles du dirigeant. Elle influence également la structure du processus décisionnel: les décisions dans les entreprises de petite taille se révèlent peu programmables ou du moins peu programmées et peu formalisées; les décideurs agissent selon une démarche qui fait largement appel à l'intuition (Marchesnay, 1991, 1995; GREPME, 1997).

L'autonomie importante dont les dirigeants bénéficient dans la conduite de leur entreprise engendre néanmoins un sentiment de solitude dans l'activité décisionnelle. D'après Gumpert et Boyd (1985), les personnes qui travaillaient auparavant dans de grandes entreprises souffrent généralement davantage de cette situation, car elles ne disposent plus de l'entourage des anciens collègues et ne profitent plus de leur aide dans l'activité professionnelle. Cependant, les ressources disponibles dans les PME se révèlent davantage consacrées à l'augmentation de la

Revue internationale P.M.E., vol. 16, nº 1, 2003

(C) 2003 - Presses de l'Université du Québec

Édifice Le Delta I, 2875, boul. Laurier, bureau 450, Sainte-Foy, Québec G1V 2M2 • Tél. : (418) 657-4399 - www.puq.uquebec.ca

Tiré de : Revue internationale P.M.E., vol. $16, \mathrm{n}^{\circ} 1$, sous la direction de Pierre-André Julien. 
production et des ventes qu'au recrutement de personnes ayant des qualités de dirigeants et susceptibles d'apporter leur soutien au responsable de PME; cela étant dû à des moyens plus faibles que dans les grandes entreprises, mais également à des réticences quant au partage de l'activité de direction par le décideur.

En matière décisionnelle, les PME se différencient également des grandes entreprises par l'importance de l'engagement personnel du dirigeant et par les conséquences potentielles de la prise de décision sur la pérennité de l'organisation. Concernant l'engagement personnel, on note que comme tout chef d'entreprise, le responsable de PME engage sa propre responsabilité dans les décisions qu'il prend en ce qui concerne l'entreprise. Lorsqu'il est propriétaire, il s'expose en outre à perdre les actifs dont il dispose et cumule ainsi risque juridique et risque patrimonial (Duchéneaut, 1996). Les faits montrent d'ailleurs que les conséquences des décisions prises sont souvent plus graves dans les PME où les actions engagées ont des répercussions plus immédiates et souvent plus fondamentales que dans les grandes entreprises. Le dirigeant de PME dispose en effet d'un droit à l'erreur très limité, dans la mesure où presque toutes ses décisions ont une importance stratégique (Mahé de Boislandelle, 1994, 1996).

\subsection{Utilité de l'activité relationnelle}

Si les échanges informationnels entre le dirigeant et les membres de son entreprise sont nécessaires pour enrichir son processus décisionnel, ils se révèlent souvent insuffisants. La mondialisation de la concurrence et les phénomènes rapides d'obsolescence des produits et des services conduisent en effet le responsable de PME à rechercher des informations à l'extérieur de l'entreprise. Pour s'informer sur son environnement, il est amené à développer des "réseaux sociaux d'information » (Filion, 1991 ; GREPME, 1997), puis à valoriser ce « capital social $^{6}$ ».

Pour le dirigeant de PME, cette activité relationnelle (ou «réticulaire») se révèle particulièrement utile, car elle lui permet de bénéficier d'informations privilégiées en utilisant des ressources limitées (Julien et Marchesnay, 1988; Fourcade, 1991 ; Julien, 1996). On note à ce sujet que les PME ne disposent généralement pas de services spécialisés dans l'étude de l'environnement (Smeltzer, Fan et Nikolaisen, 1988), alors que ceux-ci sont plus fréquents dans les grandes organisations. La recherche d'informations est donc souvent réalisée par le dirigeant luimême (Castaldi, 1986; Cooper, Folta et Woo, 1995) et les PME sont fortement dépendantes de lui à cet égard. Plusieurs études montrent d'ailleurs que les dirigeants de PME consacrent environ la moitié de leur temps à des personnes

6. L'importance du «capital social» a été soulignée par de nombreux auteurs, notamment par Bourdieu (1980), Coleman (1990), Burt (1992), Walker et al. (1997). 
extérieures à leur entreprise : amis, clients, fournisseurs, experts (juristes, expertscomptables, etc.), banquiers, autres dirigeants, membres d'organisations professionnelles, etc. (Fallery, 1983; Kurke et Aldrich, 1983). Ces liens permettent de recueillir des informations variées (Granovetter, 1973; Aldrich et Zimmer, 1986) dont l'apport peut être considéré comme «stratégique » étant donné la richesse des ressources obtenues (Falemo, 1989). Le réseau de relations ainsi créé peut alors constituer un élément majeur dans l'évolution de la vision du dirigeant dont le rôle consiste précisément à développer cette dernière pour orienter l'entreprise (Filion, 1991).

\subsection{Positionnement de l'association de dirigeants}

Parmi la multitude des sources d'aide à la décision que les responsables de PME peuvent mobiliser, quelle est la place occupée par l'association de dirigeants? Nous considérons pour cela ses trois caractéristiques en matière de mission, de cible et de formalisation en les comparant à d'autres sources potentiellement mobilisables.

\subsubsection{La mission}

La mission de l'association est orientée vers une réflexion et une action sur la gestion de l'entreprise. Dans cette optique, l'association met en relation des responsables d'entreprise les uns avec les autres, parfois en présence d'un spécialiste du sujet choisi, afin de les faire discuter ensemble sur des thèmes susceptibles de leur apporter une aide dans leur activité de direction.

Cette mission distingue l'association de dirigeants d'autres organismes tels que, par exemple, les chambres de commerce et d'industrie et les syndicats professionnels. En effet, la mission des CCI consiste davantage à augmenter la représentativité des chefs d'entreprise face aux pouvoirs publics qu'à leur permettre d'échanger leurs points de vue sur des questions liées à l'activité professionnelle. De la même façon, la mission des syndicats professionnels repose essentiellement sur la défense des intérêts des adhérents. Si ces organismes peuvent éventuellement offrir des services annexes à leur mission d'origine et proches de ceux des associations de dirigeants (par exemple des conférences animées par des spécialistes ou bien l'accès à des interlocuteurs privilégiés), leur structure tout autant que leur fonctionnement ne leur permettent généralement pas de proposer la gamme de services des associations étudiées ici (voir partie empirique). Plus largement, les réunions organisées dans d'autres types de réseaux qui rassemblent des dirigeants (associations sportives, culturelles, «clubs services » à visée humanitaire, etc.) peuvent également offrir des lieux d'échanges d'informations utiles pour les décideurs. Cependant, ces échanges ne constituent pas l'objet explicite des rencontres et ne correspondent pas à la mission de ces structures.

Revue internationale P.M.E., vol. 16, nº 1, 2003

(C) 2003 - Presses de l'Université du Québec

Édifice Le Delta I, 2875, boul. Laurier, bureau 450, Sainte-Foy, Québec G1V 2M2 - Tél. : (418) 657-4399 - www.puq.uquebec.ca

Tiré de : Revue internationale P.M.E., vol. $16, \mathrm{n}^{\circ} 1$, sous la direction de Pierre-André Julien. 
Par comparaison, Duchéneaut (1996) explique que les associations de dirigeants (ou «clubs») «proposent davantage un "service de réseau" et/ou une réflexion sur l'entreprise, le dirigeant et son environnement» (p. 27). Le chef d'entreprise membre peut bénéficier à moindre coût d'une aide pour obtenir les informations qui l'intéressent. Cette aide peut se révéler précieuse pour le dirigeant de PME confronté à une situation de pénurie relative de ressources (financières, d'informations, de temps, d'énergie, etc.). Il est également amené à réfléchir avec ses homologues sur des questions liées à son activité professionnelle au sein de son entreprise et à l'extérieur. Si ce type d'échanges convient à la plupart des responsables d'organisation, il semble particulièrement adapté aux dirigeants de petites entreprises qui sont fortement attirés par le contact verbal et personnalisé et qui ont «besoin d'un certain "désordre" et d'un réseau de communication complexe, toujours doublé de courts-circuits informels» (Fallery, 1983). Cette proximité avec de multiples acteurs reste d'actualité (Gélinas et al., 1996; Fallery, 2001) dans la mesure où encore aujourd'hui, les dirigeants de PME sous-utilisent les outils technologiques en particulier pour constituer un apport direct aux processus de décision (Amabile, Gadille et Meissonier, 2000).

\subsubsection{La cible}

L'association rassemble des personnes qui, toutes, dirigent une organisation. On se situe donc dans un contexte caractérisé par une certaine parité du fait de la similarité de la fonction décisionnelle exercée par les dirigeants dans leur entreprise.

Cette sélection de la cible permet à l'association d'occuper une position spécifique à l'intérieur du réseau personnel entretenu par le responsable de PME. Par comparaison avec les autres types de liens tissés avec les acteurs de son environnement, l'aide à la décision susceptible de lui être apportée par ses homologues membres de l'association repose en partie sur la convergence des préoccupations de chacun. Ils sont en effet confrontés à des problèmes similaires aux plans stratégique, tactique et opérationnel, et partagent les mêmes difficultés dans la gestion de leur entreprise. Les relations entre homologues constituent alors un moyen pertinent pour partager des informations à caractère professionnel (Filion, 1991; Chappoz, 1995) et pour profiter mutuellement des expériences de chacun (Saporta, 1986). La relation de parité facilite en effet la communication (Lincoln et Miller, 1987) et contribue à créer un soutien mutuel et une collaboration entre les acteurs (Kram et Isabella, 1985) susceptibles d'atténuer le sentiment de solitude du dirigeant de PME (Gumpert et Boyd, 1985). La parité dans les clubs d'entrepreneurs permet en outre l'apprentissage de la confiance propice aux échanges informationnels (Chappoz, 1995).

A contrario, la similarité entre les adhérents et la confiance qui s'instaure peuvent susciter des dérives potentiellement néfastes à la richesse de l'aide apportée en matière décisionnelle. Les effets éventuellement négatifs dus au conformisme 
sont généralement accentués lorsque les membres d'un groupe se ressemblent ( $c f$. notamment les travaux de Moscovici, 1998, en psychologie sociale). Ainsi, la similitude entre les participants d'un réseau et la force d'attraction ainsi créée «facilitent la communication, mais en même temps elles agissent comme des sortes de barrières qui empêchent l'introduction d'idées nouvelles » (Degenne et Forsé, 1994, p. 192). Dans ces conditions, le bénéfice de la mobilisation du réseau peut se révéler déprécié par un manque de richesse et de diversité des informations échangées.

\subsubsection{La formalisation}

Contrairement aux réseaux informels, le type de réseaux étudié ici comporte une structure associative. La formalisation en matière de structure et de fonctionnement offre un «lieu», un «milieu» ou un «espace » de rencontre (Bernoux, 1974, 1977; Filion, 1991; Chappoz, 1995). Au Québec, à propos du Groupement des chefs d'entreprise du Québec, Filion (1991) explique par exemple que ces clubs d'entrepreneurs « fournissent un milieu de rencontre où les entrepreneurs peuvent échanger, briser leur isolement, partager des idées et profiter des expériences des autres » (p. 66).

L'association prévoit également une certaine formalisation des rencontres. Les adhérents sont répartis dans des groupes qui fonctionnent suivant des règles préétablies, dont les lignes directrices sont généralement indiquées dans les statuts de l'association. Les éléments relatifs à la logistique (périodicité, horaire, lieu des rencontres, etc.) sont fixés à l'avance par les adhérents. La formalisation concerne aussi le déroulement des réunions (préparation, animation des échanges, compte rendu, etc.) dans le but de faciliter et de développer les interactions entre les membres.

Cette combinaison des trois caractéristiques relatives à la mission, à la cible et à la formalisation du réseau permet de considérer a priori l'association comme une formule particulièrement utile pour l'aide à la décision du dirigeant de PME. Deux questions successives de recherche se posent alors. La première vise à vérifier sur le terrain que l'association de dirigeants est bien perçue par les responsables de PME comme un moyen utile pour l'aide à la décision; la seconde vise à mettre en évidence les facteurs perçus par les dirigeants de PME membres comme étant favorables au succès de cette aide.

\section{Méthodologie}

Cette deuxième partie présente l'étude que nous avons menée auprès de responsables de petites entreprises membres d'associations de dirigeants situées en France et au Québec.

Revue internationale P.M.E., vol. 16, n ${ }^{\circ} 1,2003$ 


\subsection{Choix méthodologiques}

Les éléments théoriques exposés précédemment orientent en partie le choix de la méthode. La littérature scientifique sur les associations consacrées à une réflexion sur la gestion de l'entreprise est peu développée ${ }^{7}$, malgré quelques travaux récents ( $c f$. Duchéneaut, 1996). La littérature englobe fréquemment ce type d'associations à l'intérieur du champ beaucoup plus vaste de l'ensemble des différentes relations entretenues par les responsables de PME. Le manque relatif d'études sur l'aide apportée en matière décisionnelle par les associations de dirigeants nécessite la réalisation d'un travail en amont qui s'appuie sur l'étude directe du terrain.

Au-delà des résultats partiels produits jusqu'à présent, la littérature pose des questions sur les méthodes de recherche dans le domaine de l'activité relationnelle des dirigeants. À propos des réseaux au sens large, Borch et Arthur (1995) ainsi que Ozcan (1995) soulignent la nécessité de mettre en œuvre de nouvelles techniques et de proposer de nouvelles définitions pour analyser les réseaux mobilisés par les petites entreprises. Curran et al. (1993) expliquent que les recherches antérieures ont eu tendance à utiliser trop volontiers des méthodologies quantitatives. Ces auteurs souhaitent que les méthodes qualitatives soient plus employées, pour qu'une attention plus grande soit accordée au contenu des relations réticulaires en termes d'attentes, de normes, de points de vue et surtout de motivations à participer ou non au réseau.

$\mathrm{Vu}$ le caractère partiel des résultats produits par la littérature, et l'intérêt selon certains auteurs de mener des études permettant d'approfondir le sens des activités relationnelles entretenues par les dirigeants de PME, nous avons choisi une méthodologie de type qualitatif. Pour prendre en compte les spécificités de chaque situation, nous avons opté pour des études de cas (Yin, 1984) sur la base d'entretiens que nous avons menés auprès de responsables d'entreprises françaises et québécoises.

\subsection{Collecte des données}

L'étude a été réalisée auprès de propriétaires-dirigeants de petites entreprises (moins de 50 salariés), membres d'une association de dirigeants satisfaisant aux trois critères présentés précédemment en matière de mission, de cible et de formalisation.

La restriction opérée sur les entreprises de petite taille se justifie par le fait que plus l'entreprise est petite, plus l'étendue du pouvoir décisionnel du dirigeant est grande (GREPME, 1997 ; Duchéneaut, 1996). De la même façon, nous nous

7. On trouve cependant de nombreux manuels et articles de vulgarisation sur le sujet.

Revue internationale P.M.E., vol. 16, nº 1, 2003 
sommes intéressée aux propriétaires-dirigeants, dont le pouvoir en matière décisionnelle est généralement étendu dans les PME en raison des parts du capital qu'ils détiennent. La combinaison de ces deux critères (taille et propriété du capital) permet en outre d'effectuer des comparaisons sur une base homogène.

Trois études de cas ont été réalisées sur la base de trois associations: le «Centre des jeunes dirigeants d'entreprise » (CJD) et «Rhodanim» en France, ainsi que le «Groupement des chefs d'entreprise du Québec » au Canada ( $c f$. présentation à l'annexe I). Comme l'explique Duchéneaut (1996), les associations étudiées ici ont chacune une identité forte et constituent bien souvent en ellesmêmes un type. L'étude de plusieurs associations se révèle donc nécessaire. L'ouverture internationale vers le Canada offre l'intérêt d'une comparaison avec des associations françaises et surtout avec Rhodanim qui s'est en partie inspirée du réseau québécois ${ }^{8}$.

Les données ont été recueillies à travers des entretiens ( $c f$. guide d'entretien à l'annexe II) que nous avons menés auprès d'adhérents de ces associations en France et au Québec. Les interviews menées auprès de 39 adhérents ont été retenues pour l'analyse. Ces entretiens semi-directifs ont permis d'aborder dans une période de temps limitée une série d'éléments préalablement définis dans le guide d'entretien. Ils se sont déroulés sur une échelle de durée allant de une heure vingt minutes à deux heures environ, soit en moyenne une heure quarante-cinq minutes. Pour répondre aux questions de recherche, les responsables d'entreprise interrogés ont été placés «en situation » par des questions directement liées à la nature de leurs relations avec l'association. Pour limiter le phénomène de rationalisation a posteriori, il a été demandé aux répondants d'illustrer leurs propos par des exemples concrets. Dans la mesure du possible, la discussion a été maintenue sur des expériences d'aide à la décision vécues par les dirigeants eux-mêmes dans un passé récent, afin d'augmenter la fidélité et la précision de l'exposé.

Cette façon de procéder impose de développer un climat de confiance avec le répondant, d'autant plus que le questionnement aborde les aspects stratégiques de son activité de responsable d'entreprise. Dans le cas des entretiens menés auprès des chefs d'entreprise québécois rencontrés plusieurs années consécutives, les relations établies au cours des précédentes visites ont favorisé la confidence. De la même manière, les échanges ont été facilités avec certains adhérents du CJD rencontrés précédemment.

8. En particulier sous l'impulsion de son fondateur Marcel Devaux pendant la seconde moitié des années 1980.

Revue internationale P.M.E., vol. 16, nº 1, 2003 


\subsection{Traitement des données}

Chaque entretien a été traité suivant la même méthode. Une «fiche de synthèse d'entretien » (Huberman et Miles, 1991) a été établie pour chacune des rencontres afin de récapituler les informations signalétiques et les principaux thèmes abordés durant la visite. Chaque retranscription a été exploitée en détail afin de recenser les thèmes dans un « dictionnaire des thèmes » permettant de favoriser la maîtrise progressive des données et d'activer le processus d'analyse (Wacheux, 1996).

Pour élaborer ce dictionnaire permettant de catégoriser les thèmes recensés, nous nous sommes attachée à respecter certaines caractéristiques des catégories définies (Bardin, 1977, 1989). Ces caractéristiques sont celles d'exhaustivité (constitution préalable d'une rubrique «divers» approfondie au cours de l'analyse), d'objectivité (absence de différences significatives entre notre travail et celui réalisé par deux autres codeurs) et de pertinence (retour au terrain). La satisfaction du critère d'exclusivité a été plus délicate, étant donné que certains éléments se rattachaient à plusieurs catégories. Ces éléments ont donc été notés dans les catégories afférentes.

La validité et la fiabilité du travail réalisé (Thiétart et al., 1999) reposent sur plusieurs éléments. La validité de la collecte des données repose sur le fait que nous avons réalisé ce travail de collecte personnellement et sur le terrain. Celle des résultats provient en partie des critères d'acceptation interne et externe (validation auprès des dirigeants et auprès de la communauté scientifique), de saturation du terrain (redondance informationnelle qui justifie l'arrêt de la collecte des données) et de complétude (disparition de contradictions internes et adéquation aux faits des explications proposées). La validité des résultats apparait cependant difficilement vérifiable et demandera à être confirmée par des recherches ultérieures. La fiabilité de la recherche repose sur le travail effectué au niveau des dictionnaires par plusieurs codeurs, sur la taille importante de l'échantillon de dirigeants d'entreprise interrogés et sur les commentaires relatifs aux résultats émis par certains d'entre eux.

\section{Résultats}

Ils expriment tout d'abord une évaluation de la pertinence de l'association concernant l'aide à la décision. Ils mettent ensuite en évidence les facteurs perçus par les dirigeants de PME membres comme étant favorables au succès de cette aide. Nous précisons que le déroulement type d'une réunion d'association comporte essentiellement le traitement d'un thème de gestion déterminé au préalable par les adhérents. Ce traitement est réalisé par les adhérents eux-mêmes ou bien par un intervenant externe qui dispose des compétences requises. Une discussion a lieu au cours ou à la suite de la présentation du thème. Des questions relatives à la vie du club sont également abordées au début ou à la fin de la rencontre.

Revue internationale P.M.E., vol. 16, no 1, 2003 


\section{1. Évaluation de la pertinence de l'association}

Les dirigeants de PME français et québécois considèrent bien l'association comme un moyen qui favorise l'aide au processus décisionnel individuel. Le détail des intérêts perçus du réseau ( $c f$. graphique 1) l'indique.

GRAPHIQUE 1

Les intérêts perçus de l'association

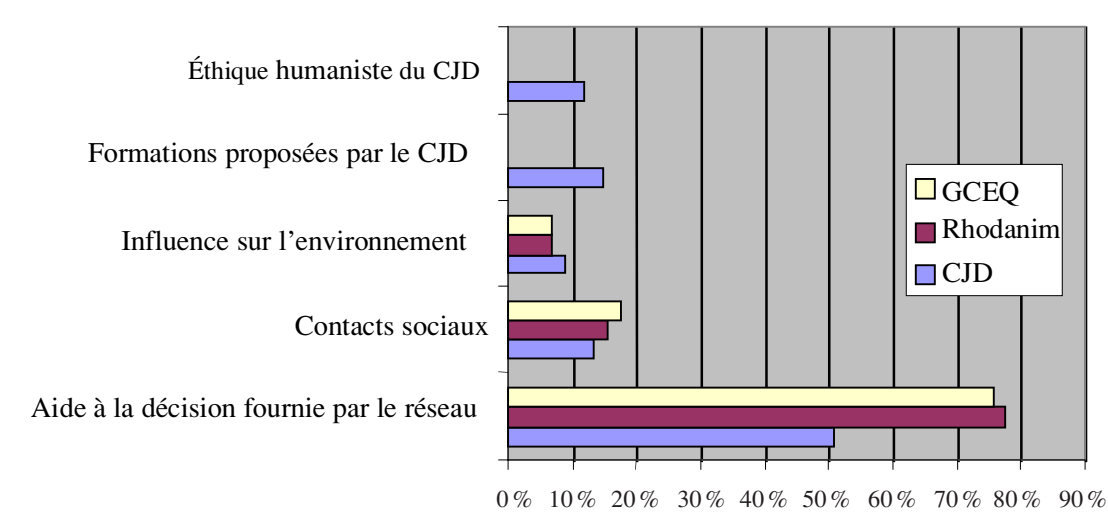

Note: La quantification des graphiques 1, 2, 3, 4 et 5 est obtenue par un comptage des récits qui correspondent au nombre de fois où chaque item apparaît dans les entretiens.

La promesse des associations, centrée sur la constitution de groupes de réflexion par des dirigeants d'entreprise sur des thèmes de gestion, en liaison avec l'environnement (institutions, organismes et spécialistes divers), est tenue. Cela explique la place prépondérante occupée par l'intérêt perçu « aide à la décision ».

L'aide apportée s'avère le plus souvent couronnée de succès ( $c f$. graphique 2 ) comme le montre l'évaluation largement positive de sa pertinence.

Cette réussite est très affirmée avec une moyenne de $87 \%$ pour les trois associations. On note que les échecs sont généralement attribués à l'absence de capacité d'aide, plutôt qu'à des intentions négatives ${ }^{9}$. L'éthique des associations fondée sur l'entraide semble donc respectée dans les faits: l'échec s'explique soit par le fait que l'aide proposée de bonne foi par les homologues ne conduit pas à des résultats jugés pertinents, soit par l'incapacité des pairs à proposer des éléments pertinents.

9. En effet: «L'information qu'ils vont te donner, ça va être celle dont ils pensent qu' elle va le plus t'aider » (GCEQ, 12, p. 4). N.B. Certaines tournures québécoises des entretiens ont été «francisées » pour en faciliter la lecture.

Revue internationale P.M.E., vol. 16, nº 1, 2003 
GRAPHIQUE 2

\section{Succès ou échec perçu de l'aide}

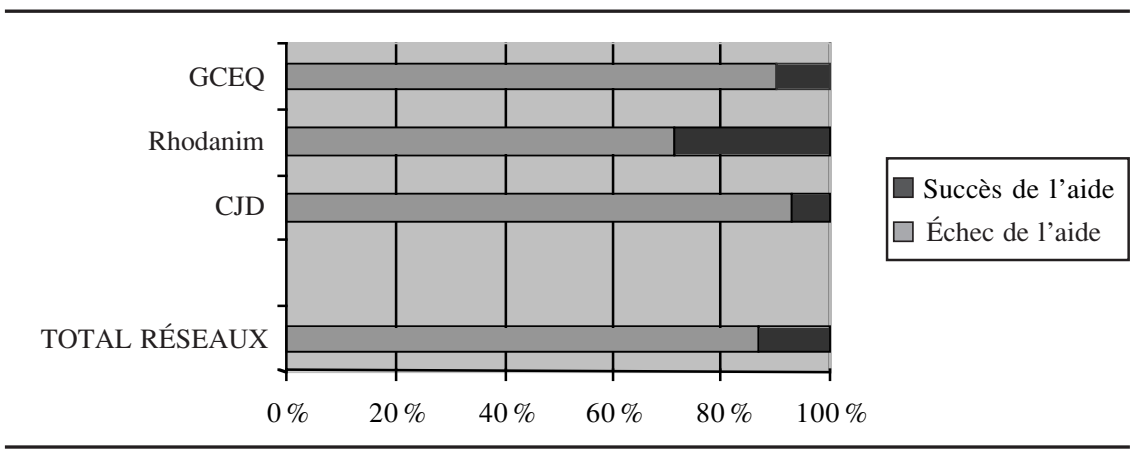

L'évaluation positive concerne néanmoins plutôt les critères d'efficience et de satisfaction que d'efficacité : l'aide apportée par l'association s'inscrit en effet davantage dans les phases en amont du processus décisionnel que dans celles situées en aval, comme l'illustre le graphique 3 ci-dessous.

GRAPHIQUE 3

\section{L'aide aux différentes phases du processus décisionnel}

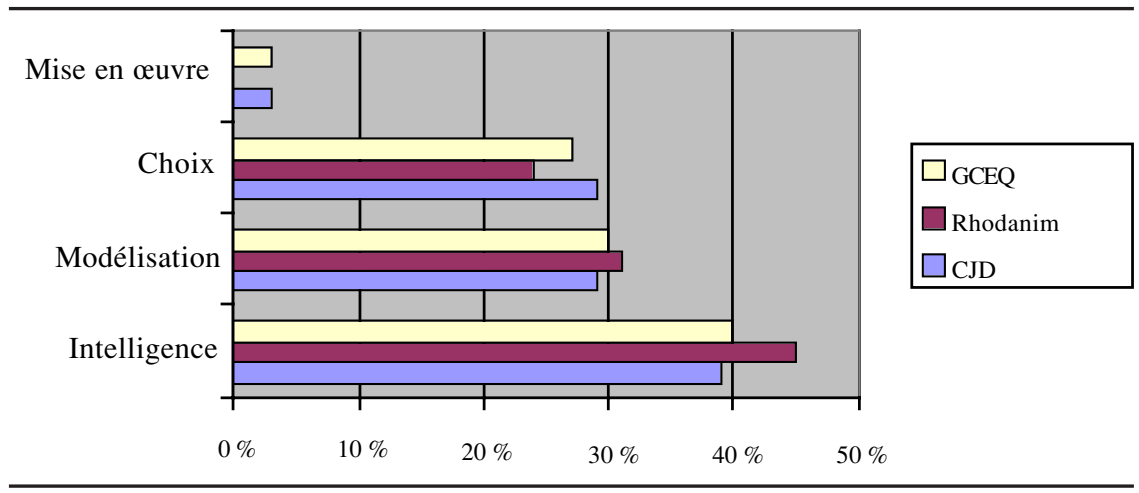

L'échange, la confrontation de points de vue, la collecte de nouvelles idées, l'expérience des homologues, sont autant d'éléments qui enrichissent le processus décisionnel dans les phases en amont, plutôt qu'en aval avec l'aide à la résolution de problèmes bien formalisés ${ }^{10}$. Cette situation se traduit par un apport au niveau

10. «Je vois le CJD comme ça: un parcours de formation où on forme plutôt l' esprit qu'un lieu où on résout des problèmes spécifiques! » $(\mathrm{CJD}, 28, \mathrm{p}$. 11.) Ou bien : «Pour l'idée, tu as besoin du Groupement, mais ce que tu vas faire avec ça, tu vas le décider toi- 
de la réflexion, plutôt qu'au niveau de l'action; l'aide étant alors évaluée davantage en termes de satisfaction et d'efficience (économie de ressources surtout temporelles, d'énergie et financières), que d'efficacité.

Les exemples moins nombreux en matière d'efficacité s'expliquent aussi par le recours des dirigeants à d'autres sources d'aide à la décision que l'association ( $c f$. graphique 4) et dont l'apport s'exprime également en aval du processus décisionnel. Parmi ces autres sources, on observe la proportion importante de récits qui mettent en scène des conseillers professionnels, le personnel de direction, les adjoints et les associés.

GRAPHIQUE 4

Sources d'aide à la décision autres que l'association

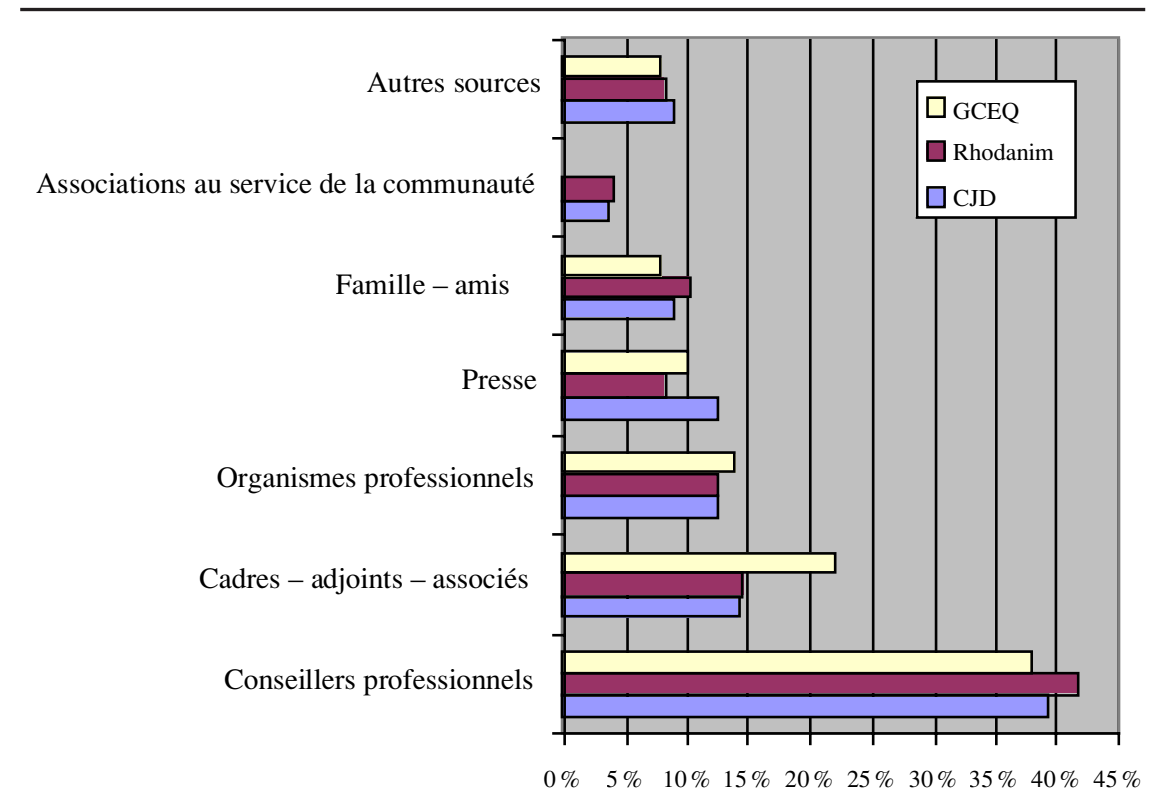

Note : En moyenne, parmi ces sources, la proportion de récits qui fait référence aux conseillers professionnels est de $40 \%$; celle des cadres, adjoints et associés, de $17 \%$.

même ou en interne » (GCEQ, 6, p. 15). Ou encore : «Il y a des gens qui sont déçus en venant au CJD parce que justement, ils trouvent qu'il y a du bla-bla. Mais c'est parce qu'ils n'ont peut-être pas compris qu'il y avait une étape avant la résolution de problèmes, c'est-à-dire qu'il faudrait savoir quels sont les problèmes qu'on va avoir demain pour essayer de s'y préparer aujourd'hui !» (CJD, 29, p. 14) 
Si l'association est complémentaire à d'autres sources, elle n'en est pas moins perçue par les dirigeants de PME comme un moyen qui occupe une place particulière. Les trois principales caractéristiques distinctives de cette catégorie de réseaux exposées dans la partie théorique (la mission, la cible et la formalisation) permettent effectivement de lui assurer un positionnement spécifique parmi les autres sources mobilisables pour l'aide à la décision :

- dans la mission, la réflexion centrée sur l'activité professionnelle dans une logique d'entraide exempte de rétribution financière constitue tout d'abord un élément distinctif ${ }^{11}$; l'assistance de l'association permet d'ailleurs de limiter le recours à des sources payantes d'aide ;

- le rassemblement de dirigeants d'entreprise constitue ensuite une cible homogène dont la similarité de préoccupations favorise la communication et la liberté de parole ${ }^{12}$. On note que la parité en climat de confiance centre l'échange informationnel sur des sujets d'ordre stratégique ${ }^{13}$ plutôt qu'opérationnel. Par ailleurs, l'absence de concurrence dans les groupes de travail, donc la diversité des horizons professionnels des adhérents, oriente l'échange vers des thèmes qui intéressent l'ensemble des membres (voir 3.2.2.);

11. «Les chefs [d'entreprise], on dit parfois de nous qu'on est juste bons à se "manger la laine sur le dos" [...] : ça veut dire manger la laine de l'autre sur son dos au lieu de marcher à côté de lui, au lieu de s'aider pour que la laine des deux pousse plus. Mais s'entraider, c' est quelque chose que j'avais originellement dans la tête et que j' approuve au Groupement, parce que c' est ce qu' on fait : en se mettant ensemble, c' est comme ça $q u^{\prime}$ on grandit. En étant ensemble avec un groupe, on peut voir plus loin que si on est tout seul. » (GCEQ, 4, p. 28)

12. «On est sûr que dans un réseau comme Rhodanim, ce sera un chef d'entreprise qui sera à côté de nous de toute façon, même s'il n'a qu'un salarié, ou même s'il en a deux cents; mais de toute façon, il y a un endroit où on est d'accord... enfin, pas forcément d'accord en termes d'accords, mais où on se reconnaît, où on a vécu la même chose » (Rhodanim, 19, p. 8). Ou bien : «Moi, je suis un gars ouvert, mais c'est sûr que je ne parlerai jamais de problèmes de financement aux gens dans ma compagnie. [...] il y a peut-être certaines choses que je vais garder plus pour mon groupe, parce que mon groupe, c'est moi dans le fond, c'est pour ça que tu n'as pas peur de parler, c'est comme si tu réfléchissais à haute voix!»(GCEQ, 10, p. 13)

13. «Je n'irai jamais voir des JD pour leur dire : j’ ai un problème opérationnel, comme par exemple mon ordinateur qui ne fonctionne pas. Si quelqu'un m'appelait pour ça, je lui dirais : débrouille-toi, je m'en fiche. Mais pour des questions un peu plus profondes, il faut les poser à quelqu' un à qui on fait confiance si c'est quelque chose d'assez sensible, et quelqu' un qui a sûrement déjà vécu une pareille situation: une mauvaise relation avec un salarié, un licenciement possible [...] Et ça aide à réfléchir.» (CJD, 28, p. 9)

Revue internationale P.M.E., vol. 16, nº 1, 2003 
- la planification des réunions, ainsi que la formalisation des rencontres, distinguent ${ }^{14}$ enfin l'association d'autres sources qui n'ont pas les mêmes éléments de structuration au sein d'un climat convivial.

Ce positionnement spécifique de l'association favorise l'optimisation des ressources du dirigeant de PME, particulièrement aux niveaux de la gestion de son temps et de l'allocation de ses ressources financières.

\subsection{Facteurs favorables au succès de l'aide}

Si l'association est bien perçue par les adhérents comme un moyen pertinent d'aide à la décision, ses caractéristiques se révèlent être particulièrement adaptées aux PME. Ces caractéristiques peuvent être classées à l'intérieur de quatre catégories :

1. la mission de l'association;

2. la structure des groupes (facteurs liés à leur composition);

3. le fonctionnement des groupes;

4. la gestion de l'association.

\subsubsection{Les facteurs relatifs à la mission}

\section{- L'orientation vers les PME}

Selon les dirigeants de PME, cette orientation contribue au succès de l'aide à la décision de ce type d'entreprises de plusieurs façons différentes et complémentaires. Elle répond d'une part au problème de la solitude décisionnelle des responsables de PME par un partage, une confrontation de leurs problèmes ${ }^{15}$ en climat de confiance, par un apaisement éventuel de leurs doutes, tout en respectant l'autonomie des entreprises adhérentes et des actions engagées par leurs dirigeants ${ }^{16}$.

14. «Eh bien, à toutes les réunions, il y a quelque chose qui se passe! On sort tout le temps avec quelque chose d'une réunion! On y va et on ne s'attend à rien; mais quand on revient, on a échangé : on a donné et on a reçu. » (GCEQ, 13, p. 9)

15. «C'est très rassurant d'avoir le témoignage des autres, c'est très important. [...] C'est un petit peu l'isolement du pouvoir quelque part et quand il y a un problème assez ardu dans votre entreprise, c'est bien de le faire partager avec des "colatéraux" [...] ou des gens qui ont été confrontés au même problème. Et là, à travers le $\mathrm{CJD}$, on trouve des solutions. Alors, je vous dis, ce n'est pas une solution style organigramme informatique : tel problème avec telle solution. C'est tout un vécu.» (CJD, 33, p. 3)

16. «Le CJD permet à des PME comme nous [...] d'être épaulées au travers d'un réseau qui, sans faire des affaires, va permettre d'avoir des réflexions que l'on aurait si on faisait partie d'un grand réseau et si on était une succursale ou une antenne. On garde notre indépendance tout en ayant une ouverture d' esprit qui est bien au-delà de sa PME.» (CJD, 39, p. 1)

Revue internationale P.M.E., vol. 16, $\mathrm{n}^{\circ}$ 1, 2003 
Elle apporte d'autre part des réponses au besoin de multicompétence des responsables de PME par la variété et la richesse ${ }^{17}$ des ressources offertes; les associations privilégient en l'occurrence le côté pratique ${ }^{18}$ et appliqué des échanges qui les aident dans leurs activités décisionnelles ${ }^{19}$.

\section{- L’incitation à l'échange de connaissances et d'expériences}

Cette mission centrée sur le partage d'informations permet aux dirigeants de PME d'enrichir leur capital social et de les aider en matière décisionnelle. On note que ce partage ${ }^{20}$ semble se réaliser de façon naturelle et spontanée en raison tout d'abord d'une sélection des postulants fondée sur leur capacité d'ouverture et de dialogue, puis de l'encouragement à ce comportement par les animateurs du réseau. Les contacts en face à face personnalisés, et souvent amicaux, conviennent aux responsables de PME pour l'aide au processus décisionnel : les connaissances et les expériences échangées constituent une base de référence suffisamment crédible pour orienter la prise de décision ${ }^{21}$. L'incitation à l'échange de connaissances et d'expériences favorise par conséquent le succès de l'aide à la décision grâce à des comportements caractérisés à la fois par une intention positive d'aide et par la mise en œuvre d'une capacité d'aide. On note que l'accès facilité des responsables de PME membres à des adhérents qui dirigent des entreprises plus importantes constitue une aide précieuse pour obtenir des informations utilisées à plus grande échelle.

17. «Le réseau, ça permet de connaître son environnement, ce qu'il y a autour de soi, savoir en permanence à qui il faut poser la bonne question. [...] C' est le carnet d' adresses et l'échange. » (Rhodanim, 19, p. 20)

18. Ces constatations s'appliquent néanmoins bien davantage à Rhodanim et au GCEQ qu'au CJD où la réflexion aborde également des aspects plus abstraits relatifs en particulier à la dimension humaniste de la gestion des entreprises.

19. «Les gens comme nous dans les petites entreprises, on n'a pas besoin de discours de grande envergure : on a besoin de parler pratique, carrément. Et le Groupement nous apporte ça. » (GCEQ, 1, p. 22)

20. «Je pense que pour ça, la structure de Rhodanim, la structure club, où il y a quand même une espèce de charte qui est reconnue, où le but du jeu, c' est la solidarité, fait en sorte aussi que ce soit normal qu'on se confie et qu'on discute de nos problèmes. » (Rhodanim, 24, p. 14)

21. «[...] dans une PME, tu ne peux pas avoir un gros conseil d'administration, donc avec des dirigeants d'entreprise [du Groupement], tu peux discuter de sujets donnés et puis tu te sers de l'expérience des autres pour prendre ta décision. »(GCEQ, 3, p. 16)

Revue internationale P.M.E., vol. 16, $\mathrm{n}^{\circ} 1,2003$ 


\section{- Le maillage avec des acteurs et avec des organismes proches des entreprises}

Ce facteur renvoie à la mission de renforcement des liens des adhérents avec divers interlocuteurs susceptibles de leur fournir une aide dans leurs activités (intervenants du monde économique, pouvoirs publics, administrations et consultants privés). Selon les dirigeants de PME, ce maillage leur permet en situation de pénurie relative de ressources d'avoir un accès privilégié à des sources d'aide à la décision adaptées $^{22}$. La sélection effectuée sur la base de la compétence et de la disponibilité des intervenants permet de favoriser le succès de l'aide à la décision des responsables de PME en climat de confiance, tout en leur permettant d'économiser des ressources.

\subsubsection{Les facteurs liés à la structure des groupes}

\section{- La taille des groupes comprise entre 7 et 12 personnes}

Cette taille ${ }^{23}$ favorise le succès de l'aide à la décision grâce à la richesse et à l'originalité des informations partagées : elle permet de maintenir en éveil l'intérêt de chacun et d'impliquer les participants ${ }^{24}$. Le climat de confiance propice aux échanges informationnels semble plus affirmé que dans un groupe plus important, en particulier pour préserver la confidentialité et la cohérence du groupe.

\section{- La stabilité de la composition des groupes}

Les dirigeants de PME estiment utile de rencontrer périodiquement les mêmes personnes pour l'aide à la décision. Les relations personnelles entre les adhérents permettent en effet de développer un échange approfondi d'informations, d'évaluer plus objectivement les forces et les faiblesses des autres adhérents et d'acquérir une connaissance étendue de la situation professionnelle des pairs. L'établissement

22. «[...] lors des assemblées générales, des colloques, il y a des spécialistes qui viennent expliquer en profondeur certains aspects : la loi du travail, la publicité [...] dans une petite entreprise, on a moins le temps que dans une grande où les gens sont spécialisés en production, en comptabilité, etc. Ici, on n'a pas le temps de vraiment s'asseoir avec un paquet de livres. Il faut connaître les nouveautés. C'est important. » (GCEQ, 7, p. 3)

23. La fourchette indiquée constitue théoriquement un groupe. L'absence d'environ $25 \%$ des membres aux réunions ramène la composition effective du groupe à six à neuf personnes.

24. «Si on est 10 ou 12 pendant une heure et demie, on peut faire le tour de table, échanger. Si on est plus, c'est déjà limite : il y a des gens qui ne parleront pas, qui ne s' investiront pas. C'est un constat qu'on a fait au cours des années. » (CJD, 32, p. 14) 
progressif d'une mémoire permet d'instaurer un climat de confiance et de proposer une aide à la décision jugée pertinente ${ }^{25}$. Cela peut constituer une réponse au droit à l'erreur minime dans les PME. La stabilité comporte néanmoins certaines limites caractérisées par un intérêt éventuellement affaibli des membres au cours du temps, une redondance informationnelle et la rareté de la nouveauté. Cette stabilité favorise donc le succès de l'aide sous réserve d'adaptations éventuelles, par exemple en matière d'ouverture à des intervenants diversifiés.

\section{- La non-concurrence}

La condition de non-concurrence au sein des groupes ${ }^{26}$ facilite la communication dans un climat de confiance ${ }^{27}$. Elle permet aux dirigeants de PME d'engager plus facilement et plus rapidement la discussion sur des points sensibles et, par conséquent, d'orienter plus volontiers la conversation vers les aspects stratégiques de leur activité. La solitude des responsables de PME peut ainsi être en partie surmontée grâce à des échanges de connaissances et d'expériences capables d'enrichir leur processus décisionnel individuel sur des questions qu'ils estiment importantes ou stratégiques. En revanche, la différence des activités exercées conduit à des échanges sur des thèmes qui concernent l'ensemble des membres et qui ne sont donc pas spécifiques à leur activité. Le détail des sujets que les adhérents choisissent d'aborder avec leurs pairs le montre (voir le graphique 5).

25. Comme l'explique un dirigeant avec un brin d'humour : «En connaissant mieux chacune des entreprises, eh bien parfois, je peux arriver six mois après et puis dire : je viens de décider de m'acheter un moteur à $20000 \$$. Connaissant la structure financière de l'entreprise, ils vont peut-être dire : tu devrais essayer d'en acheter un usagé à 10000 \$! » (GCEQ, 7, p. 23)

26. Le CJD se distingue des autres associations par le fait que les dirigeants postulants potentiellement concurrents peuvent devenir membres sous réserve d'une cooptation consensuelle. Le caractère fortement managérial des discussions traitées au CJD réduit d'ailleurs les risques éventuellement préjudiciables d'aborder des questions qui peuvent être jugées plus stratégiques, par exemple concernant le positionnement concurrentiel. La liberté de parole semble néanmoins plus grande lorsque les membres n'ont pas de concurrents dans leur section.

27. «Dans les clubs, on s'arrange justement pour que les entreprises ne soient pas concurrentes. Donc on peut très librement parler de problèmes techniques, commerciaux, etc. » (Rhodanim, 17, p. 13). 
GRAPHIQUE 5

Thèmes évoqués par les adhérents

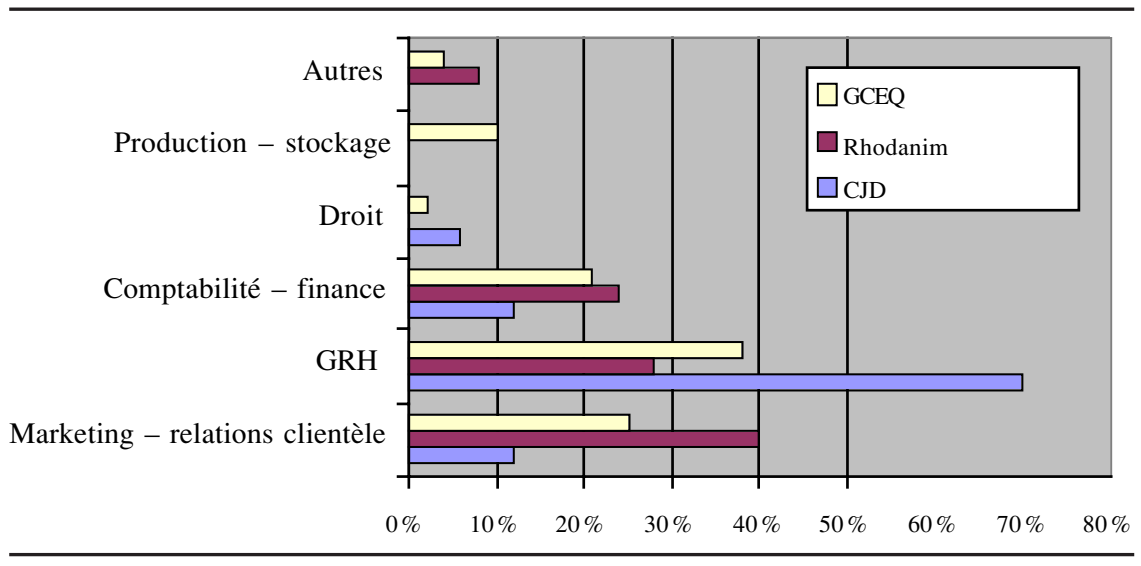

La gestion des ressources humaines tient une place prépondérante ${ }^{28}$ : elle occupe près de la moitié des sujets énoncés. Les dirigeants français et canadiens soulignent les difficultés qu'ils rencontrent dans le management des ressources humaines et le rôle clé joué par le personnel dans le développement de la PME. Les expériences vécues par les pairs dans ce domaine constituent pour eux un référentiel utile pour guider leur comportement. La condition de non-concurrence favorise par conséquent le succès de l'aide à la décision sur des thèmes qui intéressent l'ensemble des participants, mais en limite la pertinence sur des sujets liés à l'activité spécifique de chaque dirigeant.

\subsubsection{Les facteurs relatifs au fonctionnement des groupes}

\section{- La participation active des adhérents aux réunions}

La mobilisation des membres se révèle essentielle pour le succès de l'aide à la décision. Elle comprend une participation active durant la réunion, mais également aux phases antérieure et postérieure. Avant la réunion, des tâches de préparation sont nécessaires sur le fond (sélection des intervenants et travail préparatoire sur le sujet), ainsi que sur la forme (cohérence relationnelle et logistique). Le succès

28. «Essentiellement les discussions qu'on peut avoir tournent autour de l' homme dans l'entreprise » (CJD, 27, p. 12). La GRH constitue un domaine privilégié surtout au CJD et au GCEQ. Par comparaison, Rhodanim accorde une très légère avance au marketing due aux préoccupations liées aux relations avec la clientèle, notamment par le biais des réseaux de distribution.

Revue internationale P.M.E., vol. 16, nº 1, 2003 
de l'aide dépend également d'une certaine rigueur lors des réunions, particulièrement durant le tour de table ${ }^{29}$ puis pendant le traitement du sujet prévu. Une certaine structuration semble nécessaire, en particulier grâce à la présence d'un animateur formé aux techniques de conduite de réunions. L'élaboration d'un compte rendu permet finalement d'évaluer la qualité perçue de la rencontre et de réaliser un suivi entre chaque réunion. L'efficacité de la réunion est largement liée à la participation active des membres à ces différentes phases, sans pour autant négliger la convivialité et la spontanéité des échanges.

\section{- Le choix collectif des thèmes de réunion}

Cette sélection effectuée en commun par les dirigeants de PME permet de correspondre à leurs besoins spécifiques ${ }^{30}$, de susciter leur implication dans le traitement du sujet sélectionné et de favoriser le partage d'éléments capables d'enrichir leur processus décisionnel individuel.

\section{- L'assiduité aux rencontres}

Elle encourage la synergie de la communication entre les adhérents et permet de s'entraider le cas échéant. La solitude décisionnelle du dirigeant de PME trouve donc ici un palliatif. En outre, l'assiduité favorise l'adaptation aux besoins de chacun et permet de bénéficier d'informations reconnues pertinentes a posteriori ${ }^{31}$.

\section{- L'organisation des rencontres sur le lieu des entreprises des membres}

Elle offre d'une part la possibilité de bénéficier d'une visite d'entreprise commentée par son dirigeant et, par conséquent, de susciter une discussion capable d'enrichir le processus décisionnel individuel des visiteurs. D'autre part, elle permet à l'hôte d'évoquer éventuellement ses problèmes in situ et d'obtenir des commentaires et des conseils souvent pertinents ${ }^{32}$.

29. «Lui, il est passé en fin de tour de table, il n'a pas bien émis son problème, soumis son problème, évoqué son problème et on est passé au travers. » (Rhodanim, 24, p. 8)

30. «Moi, je trouve que l'organisation commerciale, pour la PME, c'est un éternel problème. Et dans notre club, on est nombreux à avoir ces problèmes-là. [...] $\mathrm{Ce}$ problème-là, on le rencontre lors des tours de table. [...] Alors on a demandé une réunion précise à ce sujet.» (Rhodanim, 24, p. 11)

31. "Quand tu as un problème, tu te dis : “oups”, c'est vrai, voilà deux mois, il s'est dit ça à telle réunion. Je ne pensais pas que j' aurais besoin de m' en servir et je m' en sers! Et voici les résultats! (GCEQ, 5, p. 6)

32. «Les équipements ne sont plus disposés comme avant suite à une visite des membres. Il y en a un qui a dit: il me semble qu'ici, si cette pièce était placée là, ce serait une 


\section{- La confidentialité}

Le respect de la confidentialité favorise le succès de l'aide à la décision, car il encourage la profondeur et la sincérité des échanges de connaissances et d'expériences en climat de confiance ${ }^{33}$. Cette contrainte de confidentialité paraît légitime à l'ensemble des dirigeants interrogés étant donné que les associations étudiées sont localisées dans des espaces géographiques restreints et que les indiscrétions commises peuvent donc parvenir très rapidement à divers acteurs tels que les concurrents par exemple.

\subsubsection{Les facteurs relatifs à la gestion des associations}

La présence de professionnels-permanents constitue une ressource appréciée par les adhérents pour l'aide à la décision. L'utilité de la "gestion de l'association par les adhérents assistés de permanents » se manifeste au regard des groupes par l'entretien d'une culture d'entraide parmi les adhérents, par la gestion des capacités des membres du groupe et par leur formation à l'animation de réunions. Par ailleurs, au Groupement des chefs d'entreprise du Québec, surtout, elle se traduit par une assistance fournie directement aux dirigeants membres ${ }^{34}$, grâce à la mise à disposition de leurs propres compétences et / ou le contact facilité à des acteurs capables de les aider.

L'action de ces permanents pallie donc dans une certaine mesure le manque relatif de ressources des dirigeants de PME et les aide pour concrétiser leurs projets.

La synthèse des différents facteurs favorables au succès de l'aide à la décision est présentée dans la figure 1 .

meilleure idée. Lui, c'est un ingénieur : il voit certaines choses. J'ai essayé ce qu'il m'a dit et puis ça fonctionne. S'il n'était pas venu chez nous, j'aurais encore le même fonctionnement. J'ai peut-être amélioré de $10 \%$ seulement, mais c'est beaucoup $10 \%$ ! On est plus efficaces, et c' est moins dangereux aussi : côté santé sécurité, c' est énorme! » (GCEQ, 5, p. 12.) Une tentative d'évaluation de l'efficacité des réunions entre celles menées dans les entreprises et celles organisées dans d'autres endroits, principalement au restaurant, aboutit à des avis partagés. Néanmoins la préférence, en matière d'aide à la décision, est généralement accordée aux rencontres dans les entreprises: outre les avantages présentés ci-dessus, ce lieu permet de mener des discussions dans un climat qui privilégie à la fois la confidentialité et le travail.

33. «J'ai confiance justement dans cette liberté de parole alliée à une certaine confidentialité. C'est là qu'on peut dire des choses sans que ce soit ni jugé, ni qu'on ait le sentiment que cela sera répété. » (Rhodanim, 17, p. 8)

34. Cette association dispose de plus de 30 permanents dont les deux tiers participent directement au développement des projets des adhérents dans leur vie professionnelle.

Revue internationale P.M.E., vol. 16, nº 1, 2003 
FIGURE 1

Les facteurs favorables au succès de l'aide à la décision

\begin{tabular}{|c|c|}
\hline $\begin{array}{l}\text { MISSION } \\
\text { DE L'ASSOCIATION } \\
\text { - Orientation vers les PME } \\
\text { - Incitation à l'échange } \\
\text { de connaissances et d'expériences } \\
\text { - Maillage avec des acteurs } \\
\text { et des organismes } \\
\text { proches des entreprises }\end{array}$ & $\begin{array}{c}\text { GESTION } \\
\text { DE L'ASSOCIATION } \\
\text { - Gestion par les adhérents assistés } \\
\text { de professionnels permanents }\end{array}$ \\
\hline & \\
\hline $\begin{array}{l}\quad \text { STRUCTURE } \\
\text { DES GROUPES } \\
\text { - Taille comprise entre } \\
7 \text { et } 12 \text { personnes } \\
\text { - Stabilité (sous réserve } \\
\text { d'adaptations) } \\
\text { - Non-concurrence }\end{array}$ & $\begin{array}{l}\text { FONCTIONNEMENT } \\
\text { DES GROUPES } \\
\text { - Participation active des adhérents } \\
\text { - Choix collectif des thèmes } \\
\text { - Assiduité } \\
\text { - Organisation des réunions sur le } \\
\text { lieu des entreprises des membres } \\
\text { - Confidentialité }\end{array}$ \\
\hline
\end{tabular}

\section{Conclusion}

Cette étude a mis en évidence le rôle joué par l'association de dirigeants dans l'aide à la décision du responsable de PME membre. Elle reconnaît à l'association un positionnement spécifique parmi les autres sources mobilisables pour l'aide, en raison de la combinaison de ses caractéristiques en matière de mission, de cible et de formalisation. L'aide apportée, qui s'appuie largement sur l'échange de connaissances et d'expériences, convient bien aux décideurs de PME qui privilégient les contacts interpersonnels directs. Elle s'exprime principalement dans les phases amont du processus décisionnel et surtout dans la phase d'intelligence, 
correspondant ainsi à un apport centré davantage sur l'efficience et la satisfaction que sur l'efficacité. La pertinence de l'aide dépend néanmoins d'un certain nombre de facteurs liés à la mission de l'association, à la structure et au fonctionnement des groupes de travail et à la gestion de l'association.

Cherchant principalement à mieux comprendre le fonctionnement de ce type de réseaux, cette étude ne prétend ni à l'exhaustivité, ni à la présentation de conclusions définitives. À l'évidence, le nombre relativement limité de personnes retenues pour l'analyse (39) ne permet tout d'abord pas de proposer des résultats immédiatement généralisables à l'ensemble des adhérents des associations étudiées, ni à toutes les associations de ce type. Ensuite, nous ne pouvons pas garantir l'exhaustivité des facteurs proposés en vue du succès de l'aide, ni expliquer de façon complète et détaillée le fonctionnement de leur processus décisionnel. La complexité de ce processus, ainsi que les limites méthodologiques de l'étude, ne nous ont permis d'appréhender ces éléments que dans leur ensemble. Enfin, nous n'avons pas examiné dans le détail les différences culturelles entre les associations françaises et l'association québécoise en raison du manque d'informations approfondies sur ce thème.

Ce travail exploratoire pourrait donc être poursuivi en étudiant le développement de ces trois associations dans le temps, afin d'analyser l'évolution du rôle qu'elles jouent dans l'aide à la décision. Il serait également intéressant d'étendre ce type de recherches à d'autres associations de dirigeants ayant des finalités proches de celles que nous avons étudiées. L'étude des spécificités culturelles d'associations de dirigeants situées dans des pays différents pourrait être approfondie, ce qui permettrait notamment d'apporter aux chefs d'entreprise des éléments susceptibles de les aider à développer des relations avec leurs homologues étrangers. Plus largement, ce type d'études pourrait favoriser un maillage des associations au-delà des frontières nationales.

Le thème de la confiance peut être intégré à ces prolongements puisqu'il apparaît déjà dans ce travail comme un catalyseur des échanges entre les membres du réseau et qu'il contribue largement au succès des relations interpersonnelles. Le caractère transversal de la confiance nécessite de poursuivre des réflexions théoriques et d'expérimenter des approches méthodologiques originales. Le thème de la confiance ouvre par ailleurs des perspectives de recherches interdisciplinaires.

Ces prolongements devraient permettre d'enrichir les résultats obtenus. Le sujet présente un intérêt réel surtout pour les PME où la rapidité d'adaptation aux mutations de l'environnement grâce à l'accès à l'information constitue l'une des principales clés du succès.

Revue internationale P.M.E., vol. 16, nº 1, 2003 


\section{Notes}

L'auteure exprime ses remerciements aux deux équipes de recherche suivantes de l'Université de Montpellier en France où elle a effectué sa thèse : le CREGO ${ }^{35}$ Centre de recherche en gestion des organisations et l'ERFI ${ }^{36}$ - Équipe de recherche sur la firme et l' industrie, pour la qualité et la rigueur scientifique de ses membres et pour la pertinence de leurs commentaires. Elle remercie la FNEGE FN $^{37}$ Fondation nationale pour l'enseignement de la gestion pour l'intérêt et la richesse des programmes du CEGAG - Centre européen de formation approfondie à la gestion. L'auteure tient également à remercier les lecteurs anonymes pour la pertinence de leurs remarques et de leurs suggestions. Ses remerciements chaleureux s'adressent aussi aux 39 dirigeants de PME qui ont bien voulu lui consacrer du temps pour répondre à son guide d'entretien et tout spécialement aux Québécois pour la gentillesse particulière de leur accueil au cours des trois séjours de recherche effectués au Québec.

ANNEXE I

Caractéristiques des associations retenues pour l'étude

\begin{tabular}{lccc}
\hline ASSOCIATIONS & $\begin{array}{c}\text { Centre } \\
\text { des jeunes } \\
\text { dirigeants } \\
\text { (CJD) }\end{array}$ & Rhodanim & $\begin{array}{c}\text { Groupement } \\
\text { des chefs } \\
\text { d'entreprise } \\
\text { du Québec }\end{array}$ \\
\hline Date de création & 1938 & 1976 & 1974 \\
\hline Nombre d'adhérents & 2100 & 70 & 800 \\
\hline $\begin{array}{l}\text { Nombre de } \\
\text { sections / de clubs }\end{array}$ & 98 sections & 6 clubs & 116 clubs \\
\hline $\begin{array}{l}\text { Périodicité } \\
\text { des rencontres }\end{array}$ & $\begin{array}{c}\text { Mensuelle } \\
\text { ou bimensuelle } \\
\text { (en fonction des } \\
\text { formations notamment) }\end{array}$ & Mensuelle & Mensuelle \\
\hline $\begin{array}{l}\text { Nombre de permanents } \\
\text { Nombre de permanents } \\
\text { par adhérent }\end{array}$ & 12 & 1 & 32 \\
\hline
\end{tabular}

35. Adresse : Université de Montpellier II - IAE, CREGO, place Eugène-Bataillon, 34095 Montpellier Cedex 5, France.

36. Adresse : Université de Montpellier I - ISEM, ERFI, avenue de la Mer, Espace Richter, Bât. E, B.P.9659, 34054 Montpellier Cedex 1, France. http ://www.erfi.univmontp1.fr

37. Adresse : FNEGE, 2, avenue Hoche, 75008 Paris, France. http ://www.fnege.fr 
AnNeXe II

\section{Guide d'entretien}

Première partie: Introduction par des questions signalétiques (10 à 15 minutes) :

- Présentation générale de l'entreprise

- Profil, parcours et pouvoir décisionnel du dirigeant

$\Rightarrow$ Objectif: Valider le pouvoir décisionnel du dirigeant et situer les exemples évoqués dans la partie 3

Deuxième partie: Transition par des questions concernant ses liens avec l'association (5 à 10 minutes) :

- Ancienneté dans l'association

- Motivations de l'adhésion

- Précisions sur les personnes qui l'ont introduit dans le réseau

- Responsabilités éventuelles dans l'association

- Assiduité aux réunions

- Intentions quant au renouvellement de l'adhésion

$\Rightarrow$ Objectif: Déterminer l'intérêt de l'adhérent pour l'association.

Troisième partie: Exposé d'exemples vécus en matière d'aide à la décision fournie par l'association (1 heure à 1 heure 30) :

- Évocation d'exemples avec demandes de précisions :

- Qui?

- Pourquoi?

- Quand?

- Où?

- Comment?

- Comparaison avec les autres sources d'aide à la décision qu'il mobilise

$\Rightarrow$ Objectif: Déterminer le rôle joué par l'association dans l' aide à la décision et faire émerger les facteurs favorables au succès de l'aide.

Quatrième partie: Propositions d'amélioration de l'offre (5 à 10 minutes), en fonction du temps restant dont dispose le répondant:

- Éléments à améliorer dans l'offre de l'association

$\Rightarrow$ Objectif: Réaliser une synthèse des éléments positifs et négatifs perçus par l'adhérent au regard de l'association pour améliorer l'offre.

\section{Bibliographie}

ALDRICH, H. et C. ZIMMER (1986), «Entrepreneurship through social networks », dans D. Sexton et R. Smilor (dir.), The Art and Science of Entrepreneurship, New York, Ballinger.

AMABILE, S., M. GAdiLlE et R. MeISSONIER (2000), «Information, organisation, décision : étude empirique sur les apports des NTIC dans les PME "internautes" », Systèmes d'Information et Management, vol. 1, n ${ }^{\mathrm{o}}$ 5, p. 41-59.

Revue internationale P.M.E., vol. 16, $\mathrm{n}^{\circ}$ 1, 2003 
BAILlETTE, P. (2000), L'importance des activités relationnelles pour l'aide à la décision. Le cas de l'adhésion du propriétaire-dirigeant de PME à une association de dirigeants, Thèse de doctorat en sciences de gestion, Université de Montpellier II, IAE.

Bardin, L. (1977/1989), L'analyse de contenu, 2e édition, Paris, Presses universitaires de France.

BARRIOL-DOUge, N. (1997), Caractéristiques et impacts des démarches managériales collectives, Thèse de doctorat en sciences de gestion, Université de Grenoble.

BernouX, P. (1974), Les nouveaux patrons. Le Centre des Jeunes Dirigeants d'Entreprise, Paris, Les Éditions Ouvrières.

Bernoux, P. (1977), «Les mouvements patronaux: le cas du C.J.D.», Économie et Humanisme, $\mathrm{n}^{\circ} 236$, p. 37-48.

BIRLEY, S. et P. WeSTHEAD (1990), «Growth and performance contrasts between types of small firms », Strategic Management Journal, 11.

BORCH, O.J. et M.B. ARTHUR (1995), « Strategic networks among small firms : implications for strategy research methodology », Journal of Management Studies, vol. 32, no 4, p. $414-441$.

Bourdieu, P. (1980), «Le Capital Social », Actes de la recherche en sciences sociales, 3 , p. 2-3.

BurT, R.S. (1992), Structural Holes, Cambridge, Harvard University Press.

CASTALDI, R.N. (1986), An Analysis of the Work Roles of CEOs of Small Firms, Englewood Cliffs, New Jersey, Prentice-Hall.

CHAPpOZ, Y. (1995), «Le rôle des réseaux d'entrepreneurs dans les processus d'apprentissages managériaux », Communication au $\mathrm{II}^{\mathrm{e}}$ congrès international francophone de la PME.

COLEMAn, J. (1990), « Social capital in the creation of human capital », American Journal of Sociology, 94, S95-S120.

Cooper, A.C., T.B. Folta et C. Woo (1995), «Entrepreneurial information search», Journal of Business Venturing, 10, p. 107-120.

CURran, J., R. JARVIS, R.A. Blackburn et S. BlaCK (1993), «Networks and small firms : constructs, methodological strategies and some findings », International Small Business Journal, vol. 11, no 2, p. 13-25.

Curran, J. et R.A. Blackburn (1994), Small Firms and Local Economic Networks, Londres, Paul Chapman Publishing.

D’AmBoise, G. et M. MuldowneY (1988), « Management theory for small business : attempts and requirements », Academy of Management Review, vol. 13, n 2, p. 226-240.

DEGENNE, A. et M. ForSÉ (1994), Les réseaux sociaux, Paris, Armand Colin.

DevauX, M. (1985), Contribution au développement des méthodes d'apprentissage de la démarche stratégique dans les MPI, Thèse de doctorat en sciences de gestion, Université des Sciences sociales de Grenoble.

DuCHÉNEAUT, B. (1995), Enquête sur les PME françaises. Identités, contextes, chiffres, Paris, Maxima.

Revue internationale P.M.E., vol. 16, nº 1, 2003 
Duchéneaut, B. (1996) Les dirigeants de PME. Enquête, chiffres, analyses, Paris, Maxima, Laurent du Mesnil Éditeur.

FALEMO, B. (1989), « The firm's external persons : entrepreneurs or network actors?», Entrepreneurship \& Regional Development, vol.1, p. 167-177.

FALLERY, B. (1983), Le système d'information du dirigeant de petite entreprise, Thèse de doctorat en sciences de gestion, Université de Montpellier.

FALlery, B. (2001), « Nouvelles technologies et travail du dirigeant», Systèmes d'Information et Management, vol. 6, n 2, p. 81-91.

FiLION, L.J. (1991), Visions et relations : les clefs du succès de l' entrepreneur, Montréal, Les Éditions de l'Entrepreneur.

FourCade, C. (1991), Petite entreprise et développement local, Paris, Eska.

Friedlander, F. et H. PICKLE (1968), «Components of effectiveness in small organizations », Administrative Science Quarterly, vol. 13, n 2, p. 289-304.

GÉlinAs, R., A. HALley, R. JACOB et J. Drolet (1996), «Les caractéristiques et les spécificités de la PME: favorables ou défavorables au juste-à-temps? », Revue internationale PME, vol. 9, n 2, p. 81-101.

GRANOVETTER, M. (1973), « The strength of weak ties », American Journal of Sociology, 78, p. 1360-1380.

Guilhon, A. et A. HAlley (1996), «Efficience logistique et stratégie », Communication au III ${ }^{\mathrm{e}}$ congrès international francophone de la PME, Université du Québec à Trois-Rivières, 23-25 octobre.

GuMPERT, D.E. et D.P. BOYD (1984), «The loneliness of the small-business owner» Harvard Business Review, novembre-décembre, p. 18-24.

GUMPERT, D.E. et D.P. BOYD (1985), «Stress et solitude du patron de PME », HarvardL'Expansion, p. 44-57.

Huberman, A.M. et M.B. Miles (1991), Analyse des données qualitatives, Bruxelles, De Boeck-Wesmael.

JULIEN, P.-A. (1996), «Le contrôle de l'information "riche" par les réseaux : clef du dynamisme des $\mathrm{PME} »$, Communication au $\mathrm{III}^{\mathrm{e}}$ congrès international francophone de la PME (CIFPME 96), Université du Québec à Trois-Rivières.

JULIEN, P.-A. (dir.) - GREPME (1997), Les PME, bilan et perspectives, $2^{\mathrm{e}}$ édition, Paris, Economica.

Julien, P.-A. et M. Marchesnay (1988), La petite entreprise, Paris, Vuibert Gestion.

KoENIG, G. (1996), Management stratégique. Paradoxes, interactions et apprentissages, Paris, Nathan.

KRAM, K.E. et L.A. IsABELla (1985), «Mentoring alternatives: the role of peer relationships in career development », Academy of Management Journal, vol. 28, $\mathrm{n}^{\mathrm{o}} 1$, p. 110-132.

KURKE, L.B. et H.E. ALDRICH (1983), « Mintzberg was right ! : a replication and extension of the nature of managerial work», Management Science, vol. 29, nº 8, p. 975-984.

Revue internationale P.M.E., vol. 16, n 1, 2003 
LinCOLN, J.R. et J. MilleR (1987), «Work and friendship ties in organizations : a comparative analysis of relational networks », Administrative Science Quarterly, vol. 24, p. 181-199.

MAHÉ DE BOISLANDELLE, H. (1994), «Esquisse d'une théorisation de la GRH en PME », Actes du Ve congrès de l'AGRH, Montpellier.

MAHÉ DE BOISLANDELlE, H. (1996), «L'effet de grossissement chez le dirigeant de PME », Actes du III congrès international francophone sur la PME, Trois-Rivières.

Marchesnay, M. (1982), «Pour un modèle d'hypofirme», dans Entreprise et Organisation, Mélanges en l'honneur de Madame le Professeur J. Aubert-Krier, Paris, Economica.

MARCheSNAY, M. (1988), La stratégie, Paris, Éditions Chotard.

MarCheSnay, M. (1991), «Mintzberg on PME», Revue internationale PME, vol. 4, nº 1.

MARCHESNAY, M. (1995), «Information, risque et systèmes de gestion de l'hypofirme », Communication au $\mathrm{II}^{\mathrm{e}}$ congrès international francophone de la PME, Paris, 25-27 octobre.

Marty, A. et G. Ivanoff (1996), Cercles et réseaux d'influence, Paris, Les Presses du Management.

MintZBERG, H., D. RAISINGHANI et A. TheORET (1976), « The Structure of "Unstructured" Decision Process », Administrative Science Quarterly, vol. 21, p. 246-275.

Moscovici, S. (1998), Psychologie sociale, Paris, Presses universitaires de France.

NUTT, P.C. (1993a), « The identification of solution ideas during organizational decisionmaking », Management Science, vol. 39, n 9, p. 1071-1085.

NuTT, P.C. (1993b), « The formulation processes and tactics used in organizational decision making », Organization Science, vol. 4, n 2, p. 226-251.

OzCAN, G.B. (1995), « Small business networks and local ties in Turkey », Entrepreneurship \& Regional Development, vol. 7, p. 265-282.

PATUREL, R. (2000), «Externalisation et entrepreneuriat», dans Histoire d' entreprendre, T. Verstraete (dir.), Paris, Éditions Management \& Société, p. 173-186.

REIX, R. (1995), Systèmes d' information et management des organisations, Paris, Vuibert.

SAPORTA, B. (1986), Stratégies pour la P.M.E., Paris, Entreprendre.

Simon, H.A. (1965), The Shape of Automation, New York, Harper and Row.

SMElTZER, L.R., G.L. FANN et V.N. NiKOlAisen (1988), «Environmental scanning practices in small business », Journal of Small Business Management, vol. 26, $\mathrm{n}^{\circ} 3$, p. 55-62.

THIÉTART, R.-A. et al. (1999), Méthodes de recherche en management, Paris, Dunod.

TRAHAND, J. (1999), «Aide à la décision », dans Encyclopédie de la gestion et du management, sous la dir. de R. Le Duff, Paris, Dalloz.

VAn LOYE, G. (1991), «Organisation du pouvoir et financement des PME », Direction et gestion des entreprises, $\mathrm{n}^{\circ} 132-133$.

Revue internationale P.M.E., vol. 16, $\mathrm{n}^{\circ}$ 1, 2003

(C) 2003 - Presses de l'Université du Québec

Édifice Le Delta I, 2875, boul. Laurier, bureau 450, Sainte-Foy, Québec G1V 2M2 • Tél. : (418) 657-4399 - www.puq.uquebec.ca

Tiré de : Revue internationale P.M.E., vol. 16, $\mathrm{n}^{\circ} 1$, sous la direction de Pierre-André Julien. 
WACHEUX, F. (1996), Méthodes qualitatives et recherche en gestion, Paris, Economica.

WALKer, G., B. Kogut et W. SHAN (1997), «Social capital, structural holes and the formation of an industry network », Organization Science, vol. 8, nº 2, p. 109-125.

WELSCH, H.P. et E.C. YouNG (1982), «The information source selection decision: the role of entrepreneurial personality characteristics », Journal of Small Business Management, p. 49-57.

YIN, R. (1984), Case Study Research, Design and Methods, Londres, Sage Publications.

Revue internationale P.M.E., vol. 16, $\mathrm{n}^{\circ}$ 1, 2003 